\title{
Android App of Location Awareness Using Li-Fi
}

\author{
Sergio Sandoval Reyes, Victor Hugo Herver Segura \\ Instituto Politecnico Nacional, Centro de Investigacion en Computacion, Mexico City, Mexico \\ \{sergio.sandoval9, vicherver \}@gmail.com
}

\begin{abstract}
Li-Fi is a technology that uses visible light from a Light Emitter Diode (LED) to transmit high speed data to a photo detector, which is connected to a smartphone or tablet. Location awareness refers to devices that can passively or actively determine their location with respect to a well-known location wireless communications device. In this paper we describe a $\mathrm{Li}-\mathrm{Fi}$ based application, in which a smart phone or tablet with a light sensor and within the range of a Li-Fi lamplight, immediately trigger events like displaying a picture, accessing a Web page, or playing a video.
\end{abstract}

Keywords: Visual light vommunications, Li-Fi lamps, Android.

\section{Introduction}

$\mathrm{Li}-\mathrm{Fi}$ is a term that was coined by Harald Haas $[1,2]$ derived from a technology known as visible light communication (VLC). This technology uses light from lightemitting diodes (LEDs) as a medium to deliver wirelessly high-speed communication in a similar manner to Wi-Fi [3]. VLC works by switching the current to the LEDs off and on at a very high speed, too fast to be noticed by the human eye. Direct line of sight is not necessary for Li-Fi to transmit a signal; light reflected off the walls can work as well reaching rates of about $70 \mathrm{Mbit} / \mathrm{s}$. Although both Wi-Fi and Li-Fi transmit data over the electromagnetic spectrum, Wi-Fi utilizes radio waves, whereas $\mathrm{Li}$-Fi uses visible light. Furthermore, while the bandwidth with Wi-Fi is close to full capacity, Li-Fi has almost no limitations on capacity. The visible light spectrum is 10,000 times larger than the entire radio frequency spectrum [4]. Researchers have reached data rates of over $10 \mathrm{Gbit} / \mathrm{s}$ [5].

Location awareness refers to devices that can passively or actively determine their location with respect to a well-known location wireless communications device. Location awareness enables new applications for ubiquitous computing systems and mobile phones or tablets. Such applications include the automatic reconfiguration of a computing device to suit the location in which it is currently being used. Examples include publishing special offers to potential customers who are near to the retailers [6].

This paper describes a Li-Fi based application, in which a smart phone or tablet with a light sensor and within the range of a Li-Fi lamplight, immediately trigger events like displaying a picture, accessing a Web page, or playing a video. The 
remainder of this paper is organized as follows: Section 2 presents a summary of works related to location-aware services. Section 3 describes the design of our location-aware application with Li-Fi lamps and Android phone or tablet with a light sensor. Section 4 shows the results of the implementation. Finally, our conclusions and future work are presented in Section 5.

\section{Related Work}

Several research works on VLC technologies have been proposed. The most important are described in the following.

\subsection{Leds--Leads-You-Right-To-A-Discount}

In [7, 8] ByteLight an English Company focused on using LEDs to provide highspeed data communications - the technology referred to as $\mathrm{Li}-\mathrm{Fi}$, felt their technology was better suited to helping people find their way around large indoor spaces. The idea was the following: imagine that you are in a department store that has replaced a number of its traditional lightbulbs with ByteLights. The lights, flickering faster than the eye can see, would emit a signal to passing smart phones. Your phone would read the signal through its camera, which would direct the smart phone (Figure 1), to pull up a deal offering a discount on a product on a nearby rack.

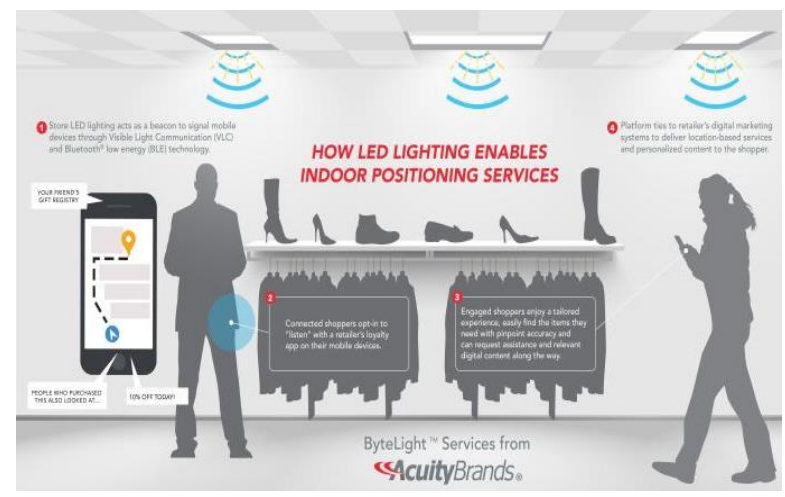

Fig. 1. A ByteLight's LED bulbs system.

\subsection{Philips Shopping Assistant With Leds And Smart Phone}

In [9] Phillips Lighting showed a LED-based indoor location detection technology. The system is similar to ByteLight, in terms of location-determination technology that relies on one-way communication between networked LED-based luminaires and customers' smartphones. In both cases the customer requires however, to download an application to utilize the technology. The communication link from luminaires to the smartphone would deliver the location data and offers. Figure 2. 


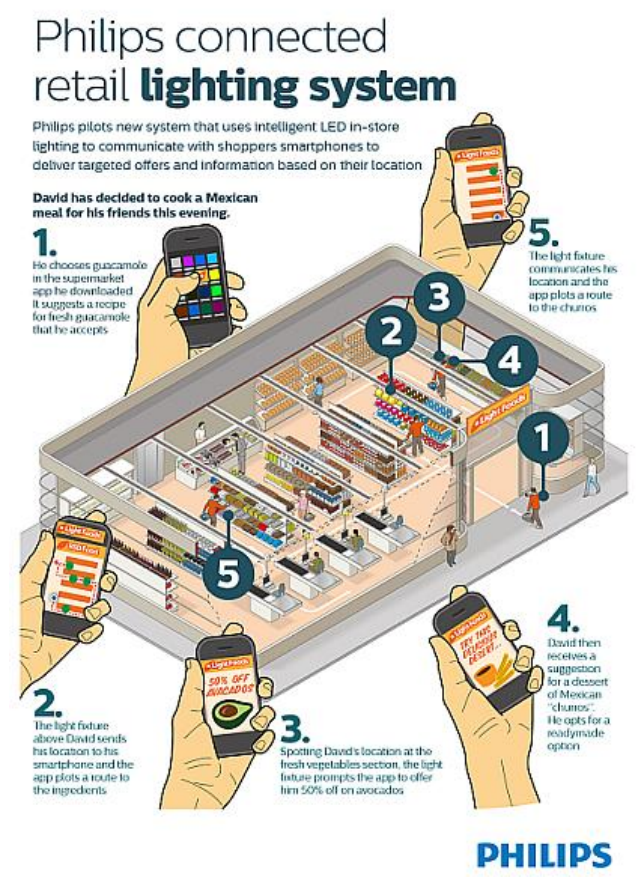

Fig. 2. Philips Lighting LED-based indoor location detection technology.

\section{$2.3 \quad$ Oledcomm Li-Fi}

Oledcomm [10] is a French Company that produces LiFi equipment in the telecom, and industrial markets, see Figure 3. It also sells kits to experiment with the Li-Fi technology.

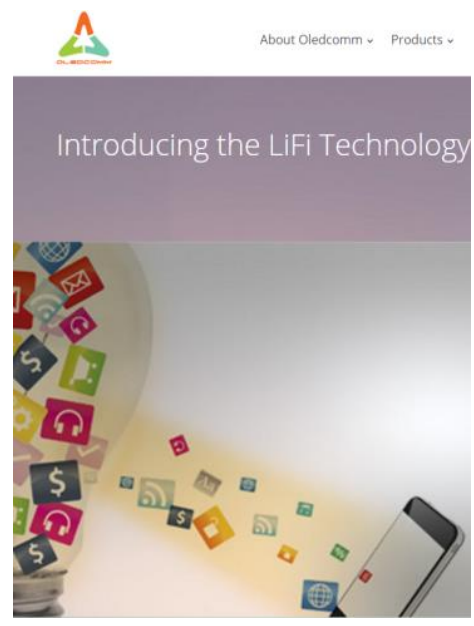

Fig. 3. Oledcomm Li-Fi. 


\section{A Location-Aware Li-Fi Application}

In the following we develop a location-aware Li-Fi application in which when an Android phone or tablet with a light sensor, is within the range of a Li-Fi lamplight, it will immediately display a picture, or access a Web page, or play a video, depending of the ID code of the lamplight. In order to do that, we will use the Oledcomm GEOLiFi XS Kit [11]. This kit contains: a) 3 GEOLiFi LED lamps, b) 1 GEOLiFi Android Tablet with an embedded sensor light; c) 1 extra GEOLiFi Dongle to be used with a Smartphone; and d) 1 GEOLiFi SDK Library. See Figure 4.

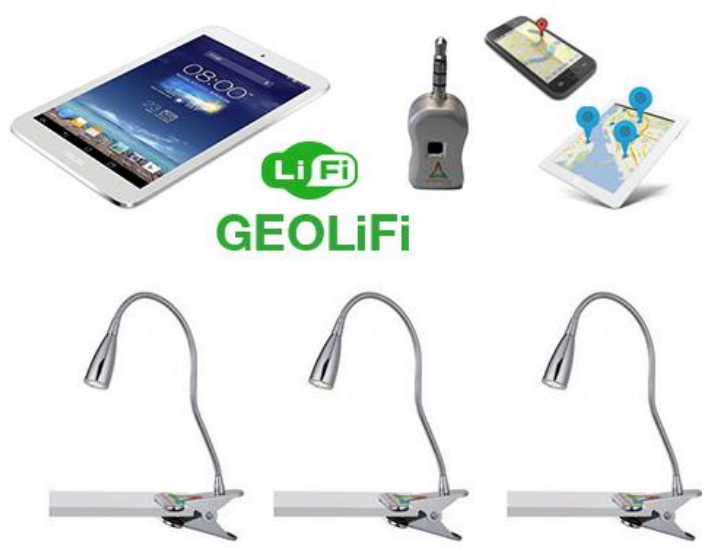

Fig. 4. The Oledcomm GEOLiFi XS Kit.

\subsection{Displaying A Picture With Lifi}

The GEOLiFi SDK Library has a small tutorial of how to use the Oledcomm GEOLiFi API. We begin loading the last version of Java SDK and Android Studio. After that we create a new Android application specifying the project and packages names with (in our case), minimum SDK 3, target SDK Android 4.4.2 and compiling with SDK 20. The most important part is in the MainActivity class in the LiFiLocation () method, Figure 5:

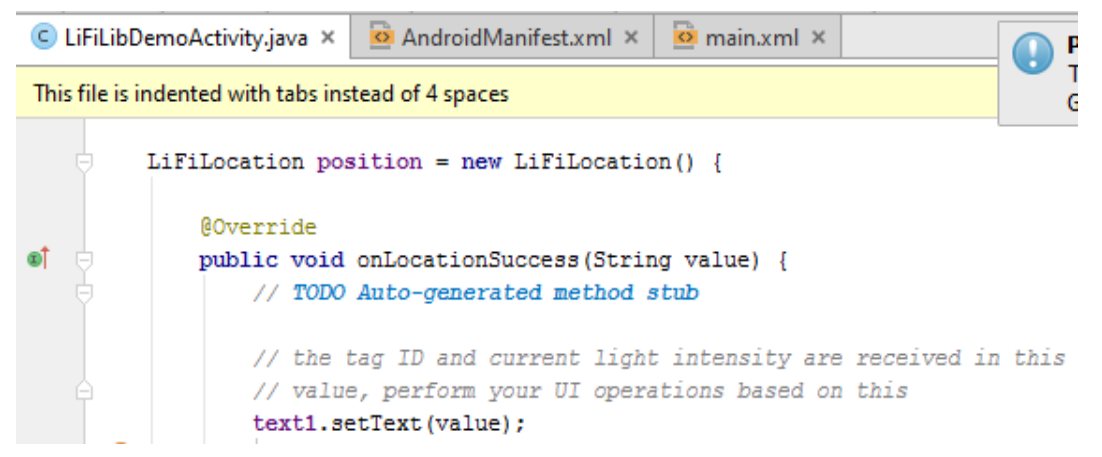

Fig. 5. The LiFiLocation () method. 
This method is used for retrieving geolocation data for a phone or tablet from a lamp Li-Fi. When this happens the corresponding Id lamp will be available. In our case, the Id of every one of the three lamps were:

a) Lamp 1: 0xd5d5

b) Lamp 2: 0x6390

c) Lamp 3: 0x6e71

Having known the Ids of the three lamps, we simply use the first Id (0xd5d5) for displaying a picture in the Tablet with the following java switch code, Figure 6:

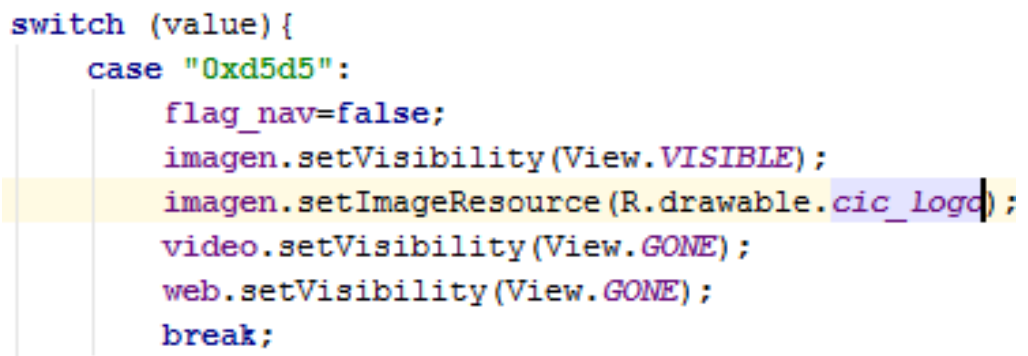

Fig. 6. Code for displaying a picture when the Tablet receives light from lamp1.

Of course, the cic_logo.png picture must be already available in the res --> drawable directory.

\subsection{Displaying A Web Page With Li-Fi}

The following java code allows access to a Web page when the tablet receives the light code (0x6390) from lamp 2. See Figure 7.

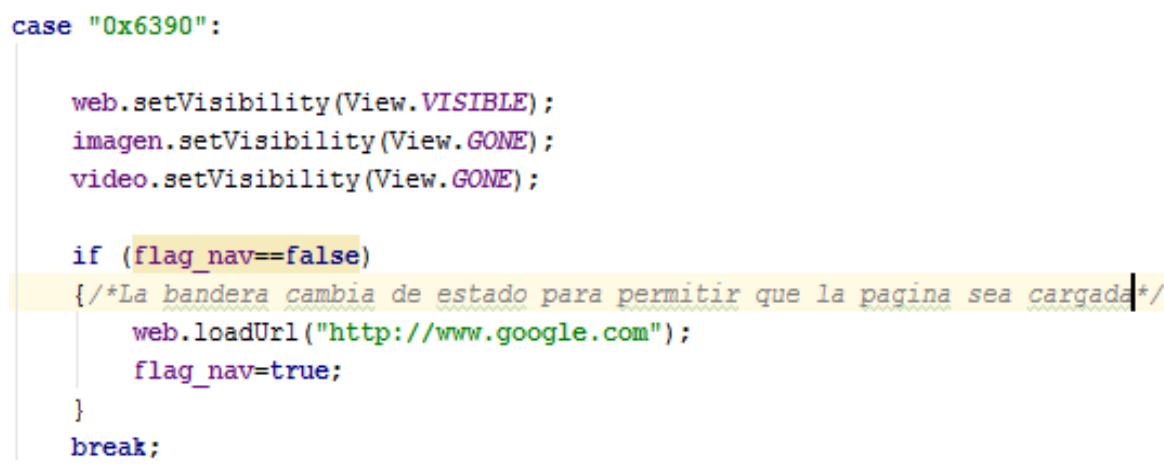

Fig. 7. Accessing a Web page when a Tablet receives light code from lamp 2.

\subsection{Displaying A Video With Li-Fi}

To display a video using $\mathrm{Li}-\mathrm{Fi}$, we use the light code $0 x 6 e 71$ and the following java code, Figure 8. The video.mp4 must be already stored in this case, in the res $\rightarrow$ raw directory. 


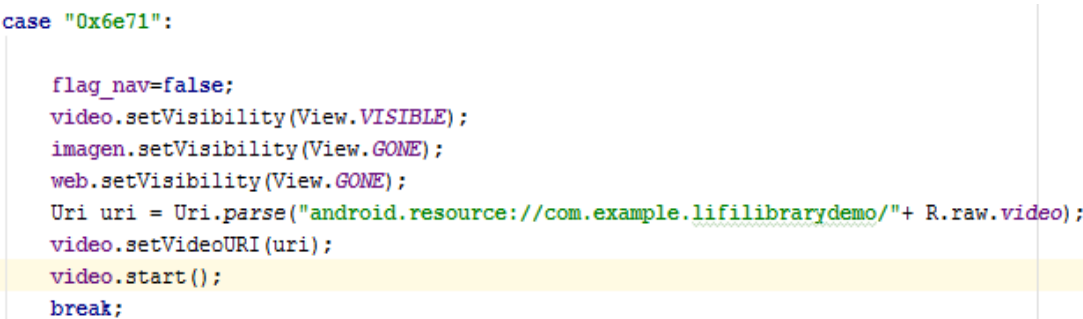

Fig. 8. Displaying a video with Li-Fi.

\section{$4 \quad$ Experiments and Results}

For the experiments we use as mentioned an Oledcomm GEOLiFi XS Kit with an Android OS 4.4.2 Tablet (Figure 9), thus as an Android 5.0 Smartphone Sony Xperia M4 Aqua, but with a light sensor connected into the earphone jack (Figure 10).

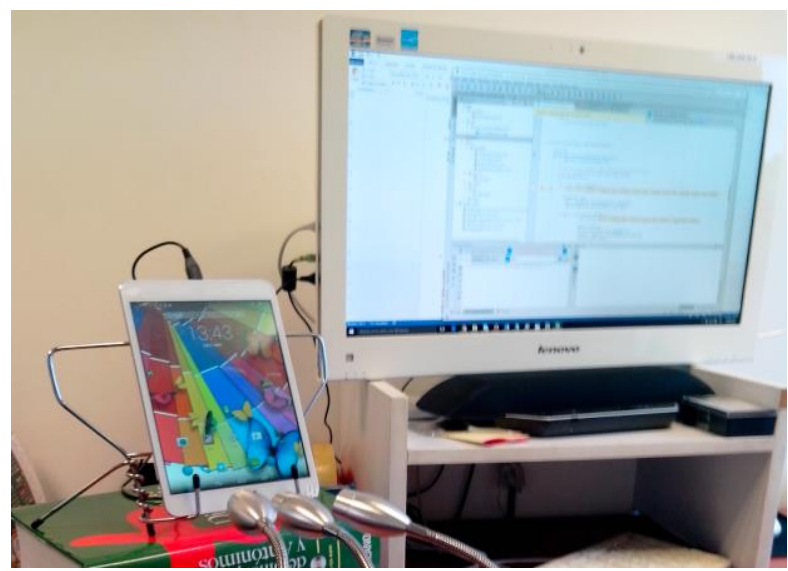

Fig. 9. GeoLiFi Kit Android Tablet 4.4.2 and three Li-Fi lamps.

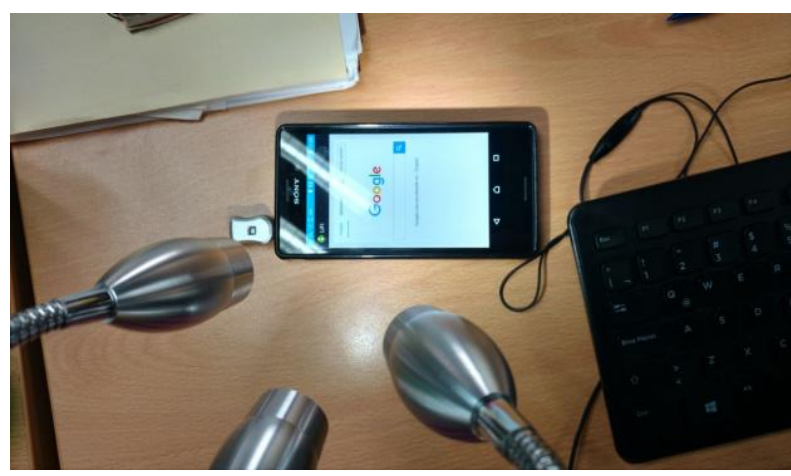

Fig. 10. Smartphone with attached Li-Fi sensor into the earphone jack. 
Figure 11 show the corresponding picture, Web page (overlapping the picture) and the video (in the upper left corner). In this same figure it can also be noticed the embedded Li-Fi sensor (down to the right from the camera lens).

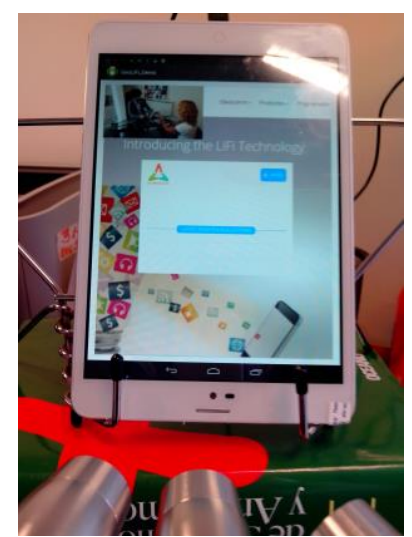

Fig. 11. Tablet with an embedded Li-Fi sensor.

\subsection{Discussion of Results}

Both devices worked fine with the Li-Fi lamps. However the smartphone with the attached Li-Fi sensor was less sensible to the Li-Fi light than the Oledcomm Tablet. The results obtained showed that indeed Li-Fi transmit data via the light from the lamps.

\section{Conclusions}

A location-aware Li-Fi application was developed using three Li-Fi lamps and two Android devices with a Li-Fi sensor. When the tablet or the smartphone was within the range of a Li-Fi lamplight, the light sensor read the code light from the lamp under test and triggered events like displaying a picture, accessing a Web page, or playing a video, according to the respective light code. This application may still be expanded beyond the actual state, making it more useful by developing for example, a mobile application to guide people through large indoor spaces like museums and stores.

Acknowledgements. This work was supported by the Instituto Politecnico Nacional Project SIP 20161439.

\section{References}

1. Harald Haas. Harald Haas: Wireless data from every light bulb. http://www.ted.com/talks/harald_haas_wireless_data_from_every_light_bulb (2016) 
2. Tsonev, Dobroslav, Videv, Stefan, Haas, Harald: Light fidelity (Li-Fi): towards all-optical networking. In: Proc. SPIE (Broadband Access Communication Technologies VIII) 9007 (2). doi:10.1117/12.2044649 (2013)

3. Sherman, Joshua: How LED Light Bulbs could replace Wi-Fi, Digital Trends. http://www.digitaltrends.com/mobile/light-bulb-li-fi-wireless-internet/ (2013)

4. Haas, Harald: High-speed wireless networking using visible light. SPIE Newsroom. doi:10.1117/2.1201304.004773; $\quad$ http://www.spie.org/newsroom/4773-high--speedwireless-networking-using-visible-light (2013)

5. Vincent, James: Li-Fi revolution: internet connections using light bulbs are 250 times faster than broadband. http://www.independent.co.uk/news/science/li-fi-revolutioninternet-connections-using-light-bulbs-are-250-times-faster-than-broadband-8909320.html (2016)

6. Wikipedia: Location awareness. https://en.wikipedia.org/wiki/Location_awareness (2016)

7. MIT Technology Review. LEDs Could Lead You Right to a Discount. https://www.technologyreview.com/s/426869/leds-could-lead-you-right-to-a-discount/ (2016)

8. AcuityBrands. ByteLight Services: Indoor positioning. http://www.acuitybrands.com/solutions/services/bytelight-services-indoor-positioning (2016)

9. IEEE Spectrum. Philips creates shopping assistant with LEDs and Smart Phone, February.http://spectrum.ieee.org/tech-talk/computing/networks/philips-creates-storeshopping-assistant-with-leds-and-smart-phone (2016)

10. Oledcomm Li-Fi. http://www.oledcomm.com/home/lifi (2016)

11. Oledcomm. GEOLiFI XS Kit. http://www.oledcomm.com/product/kit-lifi-xs (2016) 